\title{
Robust Filtering for Discrete-Time Markovian Jump Delay Systems
}

\author{
Zidong Wang, Senior Member, IEEE, James Lam, Senior Member, IEEE, and Xiaohui Liu
}

\begin{abstract}
In this letter, we study the robust filtering problem for linear uncertain discrete time-delay systems with Markovian jump parameters. The system under consideration is subjected to time-varying norm-bounded parameter uncertainties, time-delay in the state, and Markovian jump parameters in all system matrices. A filter is designed to guarantee that the dynamics of the estimation error is robustly stochastically stable in the mean square, irrespective of the admissible uncertainties as well as the time-delay. It is shown that the problem addressed can be solved in terms of the solutions to a set of coupled matrix Riccati-like inequalities.
\end{abstract}

Index Terms-Algebraic matrix inequalities, Markovian jump systems, robust filtering, time delay, uncertain systems.

\section{INTRODUCTION}

I $\mathrm{T}$ has come to be well recognized that the popular Kalman filtering theory is very sensitive to system data and has poor performance robustness when a good system model is hard to obtain or the system drifts. Various approaches have then been developed to improve the robustness of traditional Kalman filters for systems with or without time-delays, see, e.g., [2], [10].

In practice, the dynamics may experience abrupt changes in their structure and parameters caused by phenomena such as component failures or repairs changing subsystem interconnections and environmental disturbance. Such systems can be modeled as systems with Markovian jump parameters. In the past decade, the optimal regulator, controllability, observability, stability and stabilization problems have been extensively studied for jump linear systems, see [4] and references therein. The filtering problem of systems with jumping parameters has also gained much attention, see [8] and references therein. Furthermore, the robust Kalman filtering problem was studied in [6] for continuous-time uncertain jump systems, and the discrete-time counterpart of [6] was developed in [4], where the time-delay has not been taken into account. More recently, in [3], [5], the robust filtering problem was investigated for continuous timedelay jumping systems with norm-bounded uncertainties and polytopic uncertainties, respectively. Up to now, the robust filter

Manuscript received July 13, 2003; revised December 10, 2003. This work was supported in part by the EPSRC under Grants GR/S27658/01 and GR/R35018/01, the Nuffield Foundation under Grant NAL/00630/G, the University of Hong Kong under Grant HKU/CRCG/10203795, and the Alexander von Humboldt Foundation of Germany. The associate editor coordinating the review of this manuscript and approving it for publication was Prof. P. C. Ching.

Z. Wang and X. Liu are with the Department of Information Systems and Computing, Brunel University, Middlesex, UB8 3PH, U.K. (e-mail: Zidong.Wang@brunel.ac.uk).

J. Lam is with the Department of Mechanical Engineering, The University of Hong Kong, Hong Kong.

Digital Object Identifier 10.1109/LSP.2004.831729 design problem for uncertain discrete time-delay systems with Markovian jump parameters has not yet been fully investigated.

It is, therefore, our interest in this letter to tackle the filtering problem for a class of discrete time-delay uncertain systems with Markovian jumping parameters. Different from the methods used in [3], [5], the parameterization approach adopted in this letter will lead to much explicit design freedom. The expected robust filters, when they exist, are usually a large set, and the freedom can be used to meet further desired performance requirements.

\section{Problem Formulation AND AsSUMPTIONS}

Let $\left\{r_{k}, k \in \mathbb{Z}\right\}$ be a time homogeneous Markov chain taking values in a finite set $\mathcal{S}=\{1,2, \ldots, s\}$, with transition probability from mode $i$ at time $k$ to mode $j$ at time $k+1, i, j \in \mathcal{S}$

$$
p_{i j}=\operatorname{Prob}\left(r_{k+1}=j \mid r_{k}=i\right)
$$

with $p_{i j} \geq 0$, and $\sum_{j=1}^{s} p_{i j}=1$.

Consider the following class of uncertain discrete time-delay Markovian jump systems in a fixed complete probability space $(\Omega, \mathcal{F}, P)$

$$
\begin{aligned}
x_{k+1} & =\left[A\left(r_{k}\right)+\Delta A\left(k, r_{k}\right)\right] x_{k}+A_{d}\left(r_{k}\right) x_{k-d} \\
y_{k} & =\left[C\left(r_{k}\right)+\Delta C\left(k, r_{k}\right)\right] x_{k}
\end{aligned}
$$

where $x_{k} \in \mathbb{R}^{n}$ is the state, $y_{k} \in \mathbb{R}^{p}$ is the measurement output, $d$ is an unknown positive integer time delay of the system. We assume $x_{k-d}=0$ when $k-d<0, k \in \mathbb{Z}$. For fixed system mode, $A\left(r_{k}\right), A_{d}\left(r_{k}\right), C\left(r_{k}\right)$ are known constant matrices with appropriate dimensions. $\Delta A\left(k, r_{k}\right)$ and $\Delta C\left(k, r_{k}\right)$ are real-valued matrix functions representing norm-bounded parameter uncertainties and satisfy

$$
\left[\begin{array}{l}
\Delta A\left(k, r_{k}\right) \\
\Delta C\left(k, r_{k}\right)
\end{array}\right]=\left[\begin{array}{l}
M_{1}\left(r_{k}\right) \\
M_{2}\left(r_{k}\right)
\end{array}\right] F\left(k, r_{k}\right) N\left(r_{k}\right)
$$

where for fixed system mode, $M_{1}\left(r_{k}\right), M_{2}\left(r_{k}\right)$ and $N\left(r_{k}\right)$ are known real constant matrices of appropriate dimensions which characterize how the uncertain parameter in $F\left(k, r_{k}\right)$ enters the nominal matrix $A\left(r_{k}\right)$ and $C\left(r_{k}\right)$; and $F\left(k, r_{k}\right)$ is an unknown time-varying matrix function meeting

$$
F^{T}\left(k, r_{k}\right) F\left(k, r_{k}\right) \leq I, \quad \forall k \geq 0 ; \quad r_{k}=i \in \mathcal{S} .
$$

Remark 1: The system in (2) and (3) can be used to represent many important physical systems subject to modeling error, time-delay and random failures and structural changes, see e.g., [6] and references therein.

Assumption 1: The matrix $M_{2}\left(r_{k}\right)\left(\forall r_{k}=i \in \mathcal{S}\right)$ is of full row rank.

In this letter, the linear filter under consideration is of the following structure:

$$
\hat{x}_{k+1}=G\left(r_{k}\right) \hat{x}_{k}+K\left(r_{k}\right) y_{k}
$$


where $\hat{x}_{k}$ is the state estimate, and for fixed system mode, the constant matrices $G\left(r_{k}\right)$ and $K\left(r_{k}\right)$ are filter parameters to be designed.

Notice that the set $\mathcal{S}$ comprises various operation modes of the system in (2) and (3), and the Markov chain $\left\{r_{k}, k \in \mathbb{Z}\right\}$ takes values in the finite set $\mathcal{S}=\{1,2, \ldots, s\}$. For presentation convenience, we denote

$$
\begin{aligned}
A(i) & :=A_{i}, \quad A_{d}(i):=A_{d i}, \quad C(i):=C_{i} \\
M_{1}(i) & :=M_{1 i}, \quad M_{2}(i):=M_{2 i}, \quad N(i):=N_{i} \\
\Delta A(k, i) & =\Delta A_{i k}, \quad \Delta C(k, i)=\Delta C_{i k} \\
F(k, i) & =F_{i k}, \quad G(i):=G_{i}, \quad K(i):=K_{i}, \quad i \in \mathcal{S} .
\end{aligned}
$$

Now let us work on the system mode $r_{k}=i, \forall i \in \mathcal{S}$. The error state is given by $e_{k}=x_{k}-\hat{x}_{k}$, where $\hat{x}_{k}$ is the state estimate of $x_{k}$. Then, it follows from (2), (3), and (6) that

$$
\begin{array}{r}
e_{k+1}=G_{i} e_{k}+\left[\left(A_{i}+\Delta A_{i k}\right)-K_{i}\left(C_{i}+\Delta C_{i k}\right)-G_{i}\right] x_{k} \\
+A_{d i} x_{k-d}
\end{array}
$$

Moreover, we define

$$
\begin{aligned}
\eta_{k} & :=\left[\begin{array}{l}
x_{k} \\
e_{k}
\end{array}\right], \quad A_{f i}:=\left[\begin{array}{cc}
A_{i} & 0 \\
A_{i}-G_{i}-K_{i} C_{i} & G_{i}
\end{array}\right] \\
A_{f d i} & :=\left[\begin{array}{ll}
A_{d i} & 0 \\
A_{d i} & 0
\end{array}\right], \quad M_{f i}:=\left[\begin{array}{c}
M_{1 i} \\
M_{1 i}-K_{i} M_{2 i}
\end{array}\right] \\
N_{f i} & :=\left[\begin{array}{ll}
N_{i} & 0
\end{array}\right], \quad \Delta A_{f i k}:=M_{f i} F_{i k} N_{f i} \\
A_{c f i} & =A_{f i}+\Delta A_{f i k} .
\end{aligned}
$$

Then, an augmented system can be obtained from (2)-(4) and (11) that

$$
\eta_{k+1}=A_{c f i} \eta_{k}+A_{f d i} \eta_{k-d} .
$$

Assumption 2: For all $i \leq d(i \in \mathbb{Z})$, there exists a scalar $\sigma>0$ such that $\left|\eta_{k-i}\right| \leq \sigma\left|\eta_{k}\right|$.

Assumption 2 is not restrictive since $\sigma>0$ can be chosen as a sufficiently large scalar. Now, let $\eta_{k}\left(\eta_{0}, r_{0}\right)$ denote the state trajectory from the initial data $\left(\eta_{0}, r_{0}\right)$. Clearly, the system (16) admits a trivial zero solution corresponding to the initial data $\eta_{0}=0$.

Definition 1: For the uncertain time-delay jump system (16) and every initial state $\left(\eta_{0}, r_{0}\right)$, the trivial solution is robustly stochastically stable in the mean square if the following holds:

$$
\mathbb{E}\left\{\sum_{k=0}^{\infty}\left\|\eta_{k}\left(\eta_{0}, r_{0}\right)\right\|^{2} \mid \eta_{0}, r_{0}, d\right\}<\infty .
$$

We will design a linear uncertainty-independent delay-free filter (6) for the uncertain time-delay jump system in (2) and (3). Specifically, we are interested in seeking the filter parameters, $G_{i}$ and $K_{i}$, such that for all admissible time-varying parameter uncertainties $\Delta A_{f i k}$, the augmented system (16) (and therefore the error dynamics) is robustly stochastically stable in the mean square, independent of the unknown time-delay $d$.

\section{FiLter ANALysis}

Theorem 1: Let the filter parameters $G_{i}$ and $K_{i}$ be given. If there exist a set of positive scalars $\left\{\varepsilon_{i}>0, i \in \mathcal{S}\right\}$ such that the following matrix inequalities:

$$
\begin{gathered}
-P_{i}+\varepsilon_{i}^{-1} N_{f i}^{T} N_{f i}+2 A_{f d i}^{T} \bar{P}_{i} A_{f d i}<0 \\
-0.5 \bar{P}_{i}^{-1}+A_{f i}\left(P_{i}-\varepsilon_{i}^{-1} N_{f i}^{T} N_{f i}-2 A_{f d i}^{T} \bar{P}_{i} A_{f d i}\right)^{-1} A_{f i}^{T} \\
+\varepsilon_{i} M_{f i} M_{f i}^{T}<0
\end{gathered}
$$

have positive definite solutions $P_{i}>0(i \in \mathcal{S})$ where

$$
\bar{P}_{i}=\sum_{j=1}^{s} p_{i j} P_{j}
$$

then the augmented system (16) is robustly stochastically stable in the mean square, independent of the unknown time-delay $d$.

Proof: For $r_{k}=i$, define a Lyapunov functional candidate for system (16) as

$$
V_{k}\left(\eta_{k}, i\right)=\eta_{k}^{T} P_{i} \eta_{k}+\sum_{l=k-d}^{k-1} \eta_{l}^{T} Q_{i} \eta_{l}
$$

where $P_{i}>0$ is the solution to (18), (19), and

$$
Q_{i}=2 A_{f d i}^{T} \bar{P}_{i} A_{f d i} \geq 0 .
$$

Then, one has from (16) for $r_{k}=i$ and $r_{k+1}=j$ that

$$
\begin{aligned}
\mathcal{E}\left\{V_{k+1}\right. & \left.\left(\eta_{k+1}, r_{k+1}\right) \mid \eta_{k}, r_{k}\right\}-V_{k}\left(\eta_{k}, r_{k}\right) \\
= & \eta_{k}^{T}\left(A_{c f i}^{T} \bar{P}_{i} A_{c f i}+Q_{i}-P_{i}\right) \eta_{k} \\
& +\eta_{k-d}^{T}\left(A_{f d i}^{T} \bar{P}_{i} A_{f d i}-Q_{i}\right) \eta_{k-d} \\
& +2 \eta_{k}^{T} A_{c f i}^{T} \bar{P}_{i} A_{f d i} \eta_{k-d} .
\end{aligned}
$$

It follows from

$$
\Theta \Theta^{T} \geq 0, \quad \Theta:=\eta_{k}^{T} A_{c f i}^{T} \bar{P}_{i}^{1 / 2}-\eta_{k-d}^{T} A_{f d i}^{T} \bar{P}_{i}^{1 / 2}
$$

that

$$
\begin{aligned}
& 2 \eta_{k}^{T} A_{c f i}^{T} \bar{P}_{i} A_{f d i} \eta_{k-d} \leq \eta_{k}^{T} A_{c f i}^{T} \bar{P}_{i} A_{c f i} \eta_{k} \\
&+\eta_{k-d}^{T} A_{f d i}^{T} \bar{P}_{i} A_{f d i} \eta_{k-d}
\end{aligned}
$$

From (24) and the definition of $Q_{i}$ in (22), we have

$$
\begin{aligned}
& \mathcal{E}\left\{V_{k+1}\left(\eta_{k+1}, r_{k+1}\right) \mid \eta_{k}, r_{k}\right\}-V_{k}\left(\eta_{k}, r_{k}\right) \\
& \leq \eta_{k}^{T}\left(2 A_{c f i}^{T} \bar{P}_{i} A_{c f i}-P_{i}+2 A_{f d i}^{T} \bar{P}_{i} A_{f d i}\right) \eta_{k}
\end{aligned}
$$

where $\mathcal{E}\{\cdot\}$ denotes the mathematical expectation operator.

It results from the Schur Complement Lemma that, the inequalities (18) and (19) indicate

$$
\left[\begin{array}{cc}
-0.5 \bar{P}_{i}^{-1}+\varepsilon_{i} M_{f i} M_{f i}^{T} & A_{f i} \\
A_{f i}^{T} & \Xi_{i}
\end{array}\right]<0
$$

where $\Xi_{i}:=-P_{i}+\varepsilon_{i}^{-1} N_{f i}^{T} N_{f i}+2 A_{f d i}^{T} \bar{P}_{i} A_{f d i}$.

Note that $A_{c f i}=A_{f i}+\Delta A_{f i k}, \Delta A_{f i k}:=M_{f i} F_{i k} N_{f i}$ and $F_{i k}^{T} F_{i k} \leq I$. It follows from [9] that (26) is equivalent to

$$
\begin{aligned}
\Upsilon_{i}:=\left(A_{f i}+M_{f i} F_{i k} N_{f i}\right)^{T} & \left(2 \bar{P}_{i}\right)\left(A_{f i}+M_{f i} F_{i k} N_{f i}\right) \\
& -P_{i}+2 A_{f d i}^{T} \bar{P}_{i} A_{f d i}<0 .
\end{aligned}
$$


If $\eta_{k}=0$ for some finite $k$, then it is straightforward to show that the system (16) is stochastically stable in the mean square. Assume now that $\eta_{k} \neq 0$. It follows from (25) and (27) that

$$
\mathcal{E}\left\{V_{k+1}\left(\eta_{k+1}, r_{k+1}\right) \mid \eta_{k}, r_{k}\right\}-V_{k}\left(\eta_{k}, r_{k}\right) \leq \eta_{k}^{T} \Upsilon_{i} \eta_{k}<0
$$

Note that $P_{i}>0, Q_{i} \geq 0$, and $-\Upsilon_{i}>0, \forall i \in \mathcal{S}$. Define

$$
\begin{aligned}
\bar{\eta}_{k} & =\left[\begin{array}{llll}
\eta_{k} & \eta_{k-1} & \ldots & \eta_{k-d}
\end{array}\right] \\
W_{i} & =\operatorname{diag}\left\{P_{i}, Q_{i}, \ldots, Q_{i}\right\} .
\end{aligned}
$$

Then, from Assumption 2, we have

$$
\begin{aligned}
& \frac{\mathcal{E}\left\{V_{k+1}\left(\eta_{k+1}, r_{k+1}\right) \mid \eta_{k}, r_{k}\right\}-V_{k}\left(\eta_{k}, r_{k}\right)}{V_{k}\left(\eta_{k}, r_{k}\right)} \\
& \leq \frac{\eta_{k}^{T} \Upsilon_{i} \eta_{k}}{\eta_{k}^{T} P_{i} \eta_{k}+\sum_{l=k-d}^{k-1} \eta_{l}^{T} Q_{i} \eta_{l}}=\frac{\eta_{k}^{T} \Upsilon_{i} \eta_{k}}{\bar{\eta}_{k}^{T} W_{i} \bar{\eta}_{k}} \\
& \leq-\frac{\lambda_{\min }\left(-\Upsilon_{i}\right)\left|\eta_{k}\right|^{2}}{\lambda_{\max }\left(W_{i}\right)\left|\bar{\eta}_{k}\right|^{2}} \leq-\frac{\lambda_{\min }\left(-\Upsilon_{i}\right)}{\left(1+d \sigma^{2}\right) \lambda_{\max }\left(W_{i}\right)} \\
& \leq \alpha-1
\end{aligned}
$$

where

$$
\alpha:=1-\min _{i \in \mathcal{S}}\left\{\frac{\lambda_{\min }\left(-\Upsilon_{i}\right)}{\left(1+d \sigma^{2}\right) \lambda_{\max }\left(W_{i}\right)}\right\}<1 .
$$

It also follows from (30) that

$$
\alpha \geq \frac{\mathcal{E}\left\{V_{k+1}\left(\eta_{k+1}, r_{k+1}\right) \mid \eta_{k}, r_{k}\right\}}{V_{k}\left(\eta_{k}, r_{k}\right)}>0
$$

which implies $0<\alpha<1$ and

$$
\mathcal{E}\left\{V_{k+1}\left(\eta_{k+1}, r_{k+1}\right) \mid \eta_{k}, r_{k}\right\} \leq \alpha V_{k}\left(\eta_{k}, r_{k}\right) .
$$

Then, based on the relationship (33), following the same line of the proof of Theorem 1 of [7], we can show that the augmented system (16) is robustly stochastically stable in the mean square, independent of the unknown delay $d$.

\section{FILTER SYNTHESIS}

For notational simplicity, we define

$$
\begin{aligned}
\Gamma_{i}:= & P_{1 i}-2 A_{d i}^{T}\left(\bar{P}_{1 i}+\bar{P}_{2 i}\right) A_{d i}-\varepsilon_{i}^{-1} N_{i}^{T} N_{i} \\
\widehat{A}_{i}:= & A_{i}+\varepsilon_{i} M_{1 i} M_{1 i}^{T}\left(A_{i}^{T}\right)^{-1} \Gamma_{i} \\
\widehat{C}_{i}:= & C_{i}+\varepsilon_{i} M_{2 i} M_{1 i}^{T}\left(A_{i}^{T}\right)^{-1} \Gamma_{i} \\
R_{i}:= & \varepsilon_{i} M_{2 i} M_{2 i}^{T}+\left(C_{i}-\widehat{C}_{i}\right) \Gamma_{i}^{-1}\left(C_{i}-\widehat{C}_{i}\right)^{T} \\
& +\widehat{C}_{i} P_{2 i}^{-1} \widehat{C}_{i}^{T} \\
S_{i}:= & \varepsilon_{i} M_{2 i} M_{1 i}^{T}+\left(C_{i}-\widehat{C}_{i}\right) \Gamma_{i}^{-1}\left(A_{i}-\widehat{A}_{i}\right)^{T} \\
& +\widehat{C}_{i} P_{2 i}^{-1} \widehat{A}_{i}^{T} \\
\Phi_{i}:= & \varepsilon_{i} M_{1 i} M_{1 i}^{T}+\left(A_{i}-\widehat{A}_{i}\right) \Gamma_{i}^{-1}\left(A_{i}-\widehat{A}_{i}\right)^{T} .
\end{aligned}
$$

Theorem 2: If there exist a set of positive scalars $\left\{\varepsilon_{i}>0, i \in\right.$ $\mathcal{S}\}$ such that the following sets of matrix inequalities

$$
\begin{array}{r}
-0.5 \bar{P}_{1 i}^{-1}+\varepsilon_{i} M_{1 i} M_{1 i}^{T}+A_{i} \Gamma_{i}^{-1} A_{i}^{T}<0, \\
\Psi_{i}:=-0.5 \bar{P}_{2 i}^{-1}+\widehat{A}_{i} P_{2 i}^{-1} \widehat{A}_{i}^{T}+\Phi_{i}-S_{i}^{T} R_{i}^{-1} S_{i}<0
\end{array}
$$

together with $\Gamma_{i}>0$, respectively, have positive definite solutions $P_{1 i}>0$ and $P_{2 i}>0(i \in \mathcal{S})$, where the matrices $\Gamma_{i}, \widehat{A}_{i}$,
$\widehat{C}_{i}, R_{i}, S_{i}$ and $\Phi_{i}$ are defined in (34)-(39), $\bar{P}_{1 i}=\sum_{j=1}^{s} p_{i j} P_{1 j}$ and $\bar{P}_{2 i}=\sum_{j=1}^{s} p_{i j} P_{2 j}$, then the filter (6) with parameters

$$
\begin{aligned}
K_{i} & =S_{i}^{T} R_{i}^{-1}+L_{i} U_{i} R_{i}^{-1 / 2}, \\
G_{i} & =\widehat{A}_{i}-K_{i} \widehat{C}_{i}
\end{aligned}
$$

where $U_{i} \in \mathbb{R}^{p \times p}$ is arbitrary orthogonal (i.e., $U_{i} U_{i}^{T}=I$ ), $L_{i} \in \mathbb{R}^{n \times p}$ is an arbitrary matrix meeting $\Psi_{i}+L_{i} L_{i}^{T}<0$ and $\Psi_{i}$ is defined in (41), will be such that the augmented system (16) is robustly stochastically stable in the mean square, for all admissible parameter uncertainties $\Delta A_{i k}, \Delta C_{i k}$, independent of the unknown time-delay $d$.

Proof: Set $P_{i}=\operatorname{diag}\left(P_{1 i}, P_{2 i}\right)$, where $P_{1 i} \in \mathbb{R}^{n \times n}$, $P_{2 i} \in \mathbb{R}^{n \times n}$, and then define

$$
\begin{aligned}
\Sigma_{i}= & -0.5 \bar{P}_{i}^{-1}+A_{f i}\left(P_{i}-\varepsilon_{i}^{-1} N_{f i}^{T} N_{f i}-2 A_{f d i}^{T} \bar{P}_{i} A_{f d i}\right)^{-1} \\
& \times A_{f i}^{T}+\varepsilon_{i} M_{f i} M_{f i}^{T} \\
:= & {\left[\begin{array}{ll}
\Sigma_{11 i} & \Sigma_{12 i} \\
\Sigma_{12 i}^{T} & \Sigma_{22 i}
\end{array}\right] }
\end{aligned}
$$

where

$$
\bar{P}_{i}=\operatorname{diag}\left(\sum_{j=1}^{s} p_{i j} P_{1 j}, \sum_{j=1}^{s} p_{i j} P_{2 j}\right)=\operatorname{diag}\left(\bar{P}_{1 i}, \bar{P}_{2 i}\right) .
$$

It is straightforward to verify that $\Gamma_{i}>0$ and $P_{2 i}>0$ result in the satisfaction of the condition (18). Our next goal is to show that (19) is true. We have from (44) that

$$
\begin{aligned}
\Sigma_{11 i}= & -0.5 \bar{P}_{1 i}^{-1}+\varepsilon_{i} M_{1 i} M_{1 i}^{T}+A_{i} \Gamma_{i}^{-1} A_{i}^{T} \\
\Sigma_{12 i}= & \varepsilon_{i} M_{1 i}\left(M_{1 i}-K_{i} M_{2 i}\right)^{T} \\
& +A_{i} \Gamma_{i}^{-1}\left(A_{i}-G_{i}-K_{i} C_{i}\right)^{T} \\
\Sigma_{22 i}= & -0.5 \bar{P}_{2 i}^{-1}+\varepsilon_{i}\left(M_{1 i}-K_{i} M_{2 i}\right)\left(M_{1 i}-K_{i} M_{2 i}\right)^{T} \\
& +\left(A_{i}-G_{i}-K_{i} C_{i}\right) \Gamma_{i}^{-1}\left(A_{i}-G_{i}-K_{i} C_{i}\right)^{T} \\
& +G_{i} P_{2 i}^{-1} G_{i}^{T} .
\end{aligned}
$$

It follows directly from (40) that $\Sigma_{11 i}<0$. Furthermore, substituting (43) into (46) and considering the definitions of $\widehat{A}_{i}$ and $\widehat{C}_{i}$ in (35) and (36), we obtain

$$
\begin{aligned}
\Sigma_{12 i}= & \varepsilon_{i} M_{1 i} M_{1 i}^{T}+A_{i} \Gamma_{i}^{-1} A_{i}^{T}-\left(\varepsilon_{i} M_{1 i} M_{2 i}^{T}\right. \\
& \left.+A_{i} \Gamma_{i}^{-1} C_{i}^{T}\right) K_{i}^{T}-A_{i} \Gamma_{i}^{-1}\left(\widehat{A}_{i}-K_{i} \widehat{C}_{i}\right)^{T}=0 .
\end{aligned}
$$

Next, after some tedious algebraic manipulations, in terms of the definitions of $R_{i}, S_{i}$ and $\Phi_{i}$, we have

$$
\begin{array}{r}
\Sigma_{22 i}=-0.5 \bar{P}_{2 i}^{-1}+\widehat{A}_{i} P_{2 i}^{-1} \widehat{A}_{i}^{T}+\Phi_{i}-K_{i} S_{i}-S_{i}^{T} K_{i}^{T} \\
+K_{i} R_{i} K_{i}^{T} .
\end{array}
$$

By Assumption 1, we know that $R_{i}>0$, and therefore

$$
\begin{aligned}
\Sigma_{22 i}= & -0.5 \bar{P}_{2 i}^{-1}+\widehat{A}_{i} P_{2 i}^{-1} \widehat{A}_{i}^{T}+\Phi_{i} \\
& +\left(K_{i} R_{i}^{1 / 2}-S_{i}^{T} R_{i}^{-1 / 2}\right)\left(K_{i} R_{i}^{1 / 2}-S_{i}^{T} R_{i}^{-1 / 2}\right)^{T} \\
& -S_{i}^{T} R_{i}^{-1} S_{i} .
\end{aligned}
$$

By utilizing (42) and the orthogonality of $U_{i}$, we can see that $K_{i} R_{i}^{1 / 2}-S_{i}^{T} R_{i}^{-1 / 2}=L_{i} U_{i}$ and

$$
\Sigma_{22 i}=\Psi_{i}+L_{i} L_{i}^{T}
$$




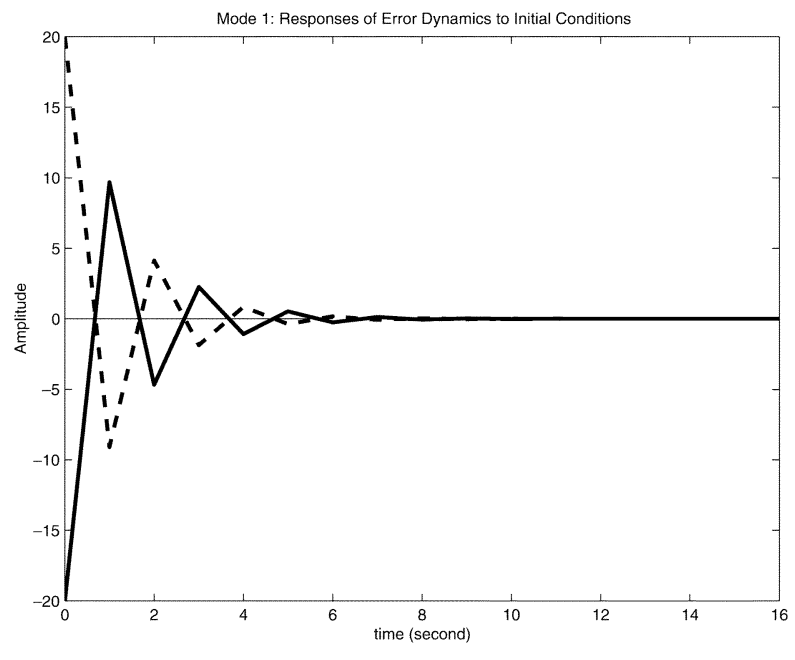

Fig. 1. $e_{1}$ (solid), $e_{2}$ (dashed).

where $\Psi_{i}$ is defined in (41). Since $L_{i} \in \mathbb{R}^{n \times p}$ is an arbitrary matrix meeting $\Psi_{i}+L_{i} L_{i}^{T}<0$, the relation $\Sigma_{22 i}<0$ follows from (51) easily. To this end, we arrive at the conclusion that $\Sigma_{i}<0$, i.e., the condition (19) is satisfied.

Finally, it follows from Theorem 1 that the augmented system (16) is robustly stochastically stable in the mean square for all admissible parameter uncertainties $\Delta A_{i k}, \Delta C_{i k}$, independent of the unknown time-delay $d$.

In the above proof, it is actually assumed that the system matrix $A_{i}$ is nonsingular. How to obtain less restrictive synthesis results would be one of our future research topics.

\section{NUMERICAL SimULATION}

Assume that the system data of (2) and (3) are as follows:

$$
\begin{aligned}
\Pi & =\left[p_{i j}\right]_{2 \times 2}=\left[\begin{array}{ll}
0.6 & 0.4 \\
0.2 & 0.8
\end{array}\right], \quad A_{1}=\left[\begin{array}{cc}
-0.3 & 0.1 \\
0.1 & 0.3
\end{array}\right] \\
A_{2} & =\left[\begin{array}{cc}
-0.5 & -0.1 \\
-0.1 & 0.6
\end{array}\right], \quad A_{d 1}=0.05 I_{2} \\
A_{d 2} & =-0.1 I_{2}, \quad C_{1}=1.2 I_{2}, \quad C_{2}=0.8 I_{2} \\
M_{11} & =0.2 I_{2}, \quad M_{12}=-0.3 I_{2}, \quad M_{21}=0.2 I_{2} \\
M_{22} & =-0.2 I_{2}, \quad N_{1}=0.08 I_{2}, \quad N_{2}=-0.1 I_{2} \\
F(k, 1) & =F(k, 2)=\sin (k) I_{2} .
\end{aligned}
$$

We choose $\varepsilon_{1}=0.1, \varepsilon_{1}=0.2$. Then, solving the Riccatilike coupled matrix inequalities (40) and (41) by the algorithm discussed in [1], we obtain

$$
\begin{aligned}
P_{11} & =\left[\begin{array}{ll}
0.0974 & 0.0006 \\
0.0006 & 0.1009
\end{array}\right] \\
P_{21} & =\left[\begin{array}{cc}
0.0948 & -0.0005 \\
-0.0005 & 0.1000
\end{array}\right] \\
P_{12} & =\left[\begin{array}{ll}
0.1938 & 0.0019 \\
0.0019 & 0.2051
\end{array}\right] \\
P_{22} & =\left[\begin{array}{cc}
0.1886 & -0.0017 \\
-0.0017 & 0.2077
\end{array}\right] .
\end{aligned}
$$

To illustrate the design flexibility, we shall make use of the freedom in selecting the parameters $L_{i}$ ( $L_{i}$ satisfies $\left.\Psi_{i}+L_{i} L_{i}^{T}<0\right)$ and $U_{i}\left(U_{i}\right.$ satisfies $\left.U_{i} U_{i}^{T} \leq I\right)$.

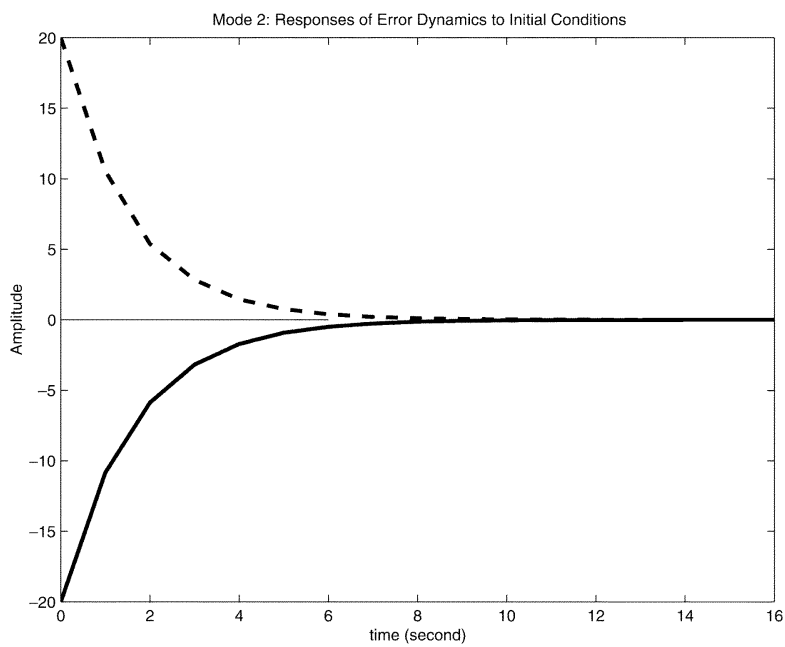

Fig. 2. $e_{1}$ (solid), $e_{2}$ (dashed).

For the system mode 1 , we choose $L_{1}=1.5 I_{2}, U_{1}=I_{2}$, and then obtain $K_{1}$ and $G_{1}$ from (42) and (43) as follows:

$$
\begin{aligned}
K_{1} & =\left[\begin{array}{ll}
0.1347 & 0.0896 \\
0.0757 & 0.6456
\end{array}\right] \\
G_{1} & =\left[\begin{array}{cc}
-0.4620 & -0.0074 \\
0.0093 & -0.4746
\end{array}\right] .
\end{aligned}
$$

We now consider the system mode 2 . In this case, we select $L_{2}=1.2 I_{2}, U_{2}=-I_{2}$ and obtain

$$
\begin{aligned}
K_{2} & =\left[\begin{array}{cc}
-1.2818 & -0.1232 \\
-0.1234 & 0.0742
\end{array}\right] \\
G_{2} & =\left[\begin{array}{cc}
0.5165 & -0.0028 \\
-0.0024 & 0.5446
\end{array}\right] .
\end{aligned}
$$

Denote the error states $e_{i}=x_{i}-\hat{x}_{i}(i=1,2)$. The responses of error dynamics to initial conditions are shown in Figs. 1 and 2 . The simulation numerical results imply that the desired goal is well achieved.

\section{REFERENCES}

[1] H. Abou-Kandil, G. Freiling, and G. Jank, "On the solution of discretetime Markovian jump linear quadratic control problems," Automatica, vol. 32, no. 5, pp. 765-768, 1995.

[2] E. K. Boukas and Z.- K. Liu, Deterministic and Stochastic Time-Delay Systems. Boston, MA: Birkhauser, 2002.

[3] E. K. Boukas and Z.-K. Liu, "Robust $H_{\infty}$ filtering for polytopic uncertain time-delay systems with Markov jumps," Comput. Elect. Eng., vol. 28, pp. 171-193, 2002.

[4] O. L. V. Costa and S. Guerra, "Robust linear filtering for discrete-time hybrid Markov linear systems," Int. J. Contr, vol. 75, pp. 712-727, 2002.

[5] M. S. Mahmoud and P. Shi, "Kalman filtering for continuous time-lag systems with Markovian jump parameters," IEEE Trans. Circuits Syst. I, vol. 50, pp. 98-105, Jan. 2003.

[6] P. Shi, E. K. Boukas, and R. K. Agarwal, "Kalman filtering for continuous-time uncertain systems with Markovian jumping parameters," IEEE Trans. Automat. Contr., vol. 44, pp. 1592-1597, Aug. 1999.

[7] — , "Control of Markovian jump discrete-time systems with norm bounded uncertainty and unknown delay," IEEE Trans. Automat. Contr., vol. 44, pp. 2139-2144, Nov. 1999.

[8] Z. Wang, J. Lam, and X. Liu, "Nonlinear filtering for state delayed systems with Markovian switching," IEEE Trans. Signal Processing, vol. 51, pp. 2321-2328, Sept. 2003.

[9] L. Xie and Y. C. Soh, "Guaranteed cost control of uncertain discrete-time systems," Control Theory Adv. Technol., vol. 10, pp. 1235-1251, 1995.

[10] X. Zhu, Y. C. Soh, and L. Xie, "Robust Kalman filters design for discrete time-delay systems," Circuits, Syst., Signal Process., vol. 21, no. 3, pp. 319-335, 2002. 